

\title{
Quantifying the motor power of trees
}

Tancrède Alméras, Barbara Ghislain, Bruno Clair, Amra Secerovic, Gilles

Pilate, Meriem Fournier

\section{To cite this version:}

Tancrède Alméras, Barbara Ghislain, Bruno Clair, Amra Secerovic, Gilles Pilate, et al.. Quantifying the motor power of trees. Trees - Structure and Function, 2018, 32 (3), pp.689-702. 10.1007/s00468018-1662-7 . hal-01897788

\section{HAL Id: hal-01897788 \\ https://hal.science/hal-01897788}

Submitted on 17 Oct 2018

HAL is a multi-disciplinary open access archive for the deposit and dissemination of scientific research documents, whether they are published or not. The documents may come from teaching and research institutions in France or abroad, or from public or private research centers.
L'archive ouverte pluridisciplinaire HAL, est destinée au dépôt et à la diffusion de documents scientifiques de niveau recherche, publiés ou non, émanant des établissements d'enseignement et de recherche français ou étrangers, des laboratoires publics ou privés. 


\title{
Quantifying the motor power of trees
}

\author{
Tancrède Alméras ${ }^{1} \mathbb{D} \cdot$ Barbara Ghislain $^{2} \cdot$ Bruno Clair $^{2} \cdot$ Amra Secerovic $^{3} \cdot$ Gilles Pilate $^{3} \cdot$ Meriem Fournier $^{4}$
}

\begin{abstract}
Trees have a motor system to enable upright growth in the field of gravity. This motor function is taken on by reaction wood, a special kind of wood that typically develops in leaning axes and generates mechanical force during its formation, curving up the stem and counteracting the effect of gravity or other mechanical disturbances. Quantifying the mechanical stress induced in wood during maturation is essential to many areas of research ranging from tree architecture to functional genomics. Here, we present a new method for quantifying wood maturation stress. It consists of tilting a tree, tying it to a stake, letting it grow in tilted position, and recording the change in stem curvature that occurs when the stem is released from the stake. A mechanical model is developed to make explicit the link between the change in curvature, maturation strain and morphological traits of the stem section. A parametric study is conducted to analyse how different parameters influence the change in curvature. This method is applied to the estimation of maturation strain in two different datasets. Results show that the method is able to detect genotypic variations in motor power expression. As predicted by the model, we observe that the change in stem curvature is correlated to stem diameter and diameter growth. In contrast, wood maturation strain is independent from these dimensional effects, and is suitable as an intrinsic parameter characterising the magnitude of the plant's gravitropic reaction.
\end{abstract}

Keywords Biomechanics $\cdot$ Gravitropism $\cdot$ Reaction wood $\cdot$ Maturation strain $\cdot$ Eccentricity $\cdot$ Efficiency

\section{Introduction}

Trees are tall slender vertical structures. Their stability is challenged by different external forces, such as wind and gravity. Their mechanical design is adapted to withstand these constraints. This is achieved for example by a strong anchorage and a stiff trunk, made of a stiff and light material,

Tancrède Alméras

tancrede.almeras@umontpellier.fr

1 LMGC, CNRS, Université de Montpellier, cc 048, Place E. Bataillon, 34095 Montpellier, France

2 CNRS, UMR EcoFoG, AgroParisTech, Cirad, INRA, Université des Antilles, Université de Guyane, 97310 Kourou, France

3 BioForA, INRA, ONF, 45075 Orléans, France

4 Université de Lorraine, AgroParisTech, INRA, UMR Silva, 54000 Nancy, France namely wood. These features are part of the tree "skeletal system" (Moulia et al. 2006), and are a necessary condition for its stability in the terrestrial environment. This is not, however, a sufficient condition. Growing straight and vertical also involves the action of a "motor system" (Moulia et al. 2006). This is related to the way of growing of the tree. The diameter growth of the stem is achieved by the addition of new wood layers at its external surface. During growth, the load applied on the tree by gravity (i.e. the self-weight) increases at the same time the structure itself increases in size. Because a tree is never perfectly symmetric, the increase in load induces a downward bending movement of the stem. Later growth increments will be added on a bent structure, acting with a larger lever arm, thus inducing further increase in bending moment and change in stem curvature. This situation would always lead to a weeping structure if the effect of gravity was not actively counteracted by the action of a motor system (Fournier et al. 2006; Alméras and Fournier 2009). 
Trees are able to induce forces in their wood, in a way similar to the muscles of animals, although the underlying mechanism differs (Alméras and Clair 2016). Normal wood induces forces of low magnitude, while large magnitude is achieved by the production of a special kind of wood, called reaction wood. This wood tends to contract (tension wood) or expend (compression wood) after it is formed. Because this dimensional change is impeded, mechanical stress is induced in wood (Archer 1986). As reaction wood is produced only either on the upper side (tension wood) or on the lower side (compression wood) of a tilted stem, the formation of a new wood layer induces asymmetric distribution of stress. This reaction is generally accompanied with eccentric growth (faster radial growth on the side with reaction wood) and changes in wood elastic properties (Alméras et al. 2005; Clair and Thibaut 2014), increasing the asymmetry of forces and thus the bending moment. This results in a bending moment, able to bend up the tree or just compensate for the effect of increasing weight (Alméras and Fournier 2009; Huang et al. 2010).

The magnitude of the mechanical stress induced in wood is quantified through the maturation strain. This parameter can be defined as the strain that would have been induced during wood formation if it were not impeded by surrounding material. Quantifying this parameter is of major importance in different kinds of studies. In forest and wood sciences, the mechanical stresses induced in wood have important economic consequences. They are cumulated over time within the stem (Archer 1986; Kubler 1987), and cause cracks and deformations when felling the tree or sawing the logs. In ecology, this parameter is important to quantify the diversity in this major function of trees (Fournier et al. 2013; Alméras et al. 2009; Clair et al. 2006) and possible trade-offs with other functions (Alméras et al. 2009). In botany, accounting for the action of tension wood is necessary to understand how the reorientation of axes in involved in the achievement of tree architecture (Fisher and Stevenson 1981). In plant physiology, this parameter is relevant to studies of gravitropism (Coutand et al. 2007; Bastien et al. 2013), i.e. the way by which plant perceive and react to gravity, to quantify the response (mechanical stress) to a stimulus (gravity). Tension wood expression has been taken as a model for functional genomics of wood formation (Pilate et al. 2004). Quantifying tension wood maturation stress is also essential to studies aiming at understanding, at molecular scale, the mechanism by which the plant is able to generate forces (as reviewed in Alméras and Clair 2016).

The usual method for quantifying maturation strain is the released strain method. It consists of releasing the stress at the tree surface and recording subsequent strains. Practically, this is done by setting a LVDT or strain gage (Kikata 1972) at the stem surface, and cutting two grooves above and below the sensor. The released strain is assumed equal to maturation strain. This assumption is based on the fact that the wood layer at the outermost surface of the stem has been deposited recently. If the trunk is stiff enough and radial growth quick enough, associated increment in external loads has induced negligible stress increment on this layer, so that it is in a nearly native state, i.e. loaded by maturation strain only. Theoretically, this would be true for any tree in active diameter growth, provided the strain is measured at the tree surface and completely released. However, practically, the operation of strain release is made with a sensor of finite length and making grooves of finite depth (Yoshida 2002). If the grooves are too shallow, not all the stress can be released so that maturation strain is underestimated. If the grooves are too deep, then older layers are also released and the mean state of stress of the probed wood layers is not native. Experiments and calculations show that, because of maturation strains and their accumulation over time, the field of stress in a tree stem has a specific shape (Kubler 1987). In particular, below the tree surface there is a stress gradient, so that the mean stress on a finite depth differs from the surface stress. Moreover, if the changes in tree self-load are quicker than the increase in diameter, as for example due to the development of branches or fruits, the increment of mechanical stress due to external action is no more negligible, so that the outer layer is not in a native state, and the released strain differs from maturation strain.

These metrological issues are particularly important when young stems or branches are studied, as is the case for most studies in a biological context (e.g. Alméras et al. 2006; Clair et al. 2011; Coutand et al. 2014, Gorschkova et al. 2015, Lafarguette et al. 2004; Nishikubo et al. 2007; Roussel and Clair 2015). For small trees, scaling effects due to allometric growth make the stem more sensitive to changes in self-weight, and the finite size of sensors make the experimental method more constraining, so that measurement of maturation strains with the released strain method fails. An alternative method has been proposed to quantify maturation strains during up-righting movement of tilted plant (Coutand et al. 2007). The method consists of tilting a plant and recording its free up-righting movement. Using a mechanical model, we (Fournier et al. 2006; Alméras and Fournier 2009) made explicit the relation between the variation in curvature (in response to the development of asymmetric maturation strains), dimensions of the section, growth rate, characteristics of the section and reaction wood maturation strain. This model can be used to deduce the maturation strain from the measured change in curvature and the morphological parameters of the stem, as done in Coutand et al. (2007) and Alméras et al. (2009). This method does not face the same metrological issues as the released strain method, and has the great advantage to be non-destructive as it can be applied during the reaction. It has however some drawbacks. The 
change in stem curvature on a finite period of time actually depends on the effect of maturation strain and the change in self-weight, and a correction has to be used to cancel the latter (Alméras et al. 2009). Moreover, another problem is that during the up-righting movement of the stem, the reaction is neither constant in space nor constant in time. Indeed, the up-righting movement is accompanied by a straightening movement (Coutand et al. 2007; Bastien et al. 2013) involving production of reaction wood on the opposite side of the distal parts of the stem (Coutand et al. 2007). Therefore, the model for computing maturation strains has to be applied locally on the stem and for small periods of time, making it heavy to use.

Here, we propose an alternative method, aiming at being more precise and less time-consuming than existing methods. The method consists in tilting a stem, fasten it to a stake, and let it grow in this constrained configuration. At the end of the experiment, the stem is released from the stake and the spring-back movement of the stem is recorded as a change in curvature. Then, a mechanical model can be used to infer the maturation strain from this change in curvature and morphological parameters of the stem (such as it size, growth rate, and eccentricity). The mathematical formulation of this model will be first shown and then used to perform a parametric study analysing the dependence between morphological parameters and change in stem curvature. To demonstrate its potential in comparison with other existing methods, this new method has been applied onto two datasets.

\section{Modelling stem reaction}

\section{Summary of this section}

In this section, we make explicit the relation between the change in curvature at the level of a stem section, the value of reaction wood maturation strain, and other parameters describing the section, such as initial diameter, diameter increment, eccentricity and variations in elastic modulus. This derivation is shown for three representations of the stem section: a general representation, a model based on sine circumferential variations of properties, and a model based on step variations in properties (as described in next section, Fig. 1). For each representation of the section, two cases will be considered. In the first case, we compute the change in curvature of the stem freely up-righting during growth. In the second case, the stem is restrained during growth by tying it to a stake, and we compute the change in curvature of the stem when releasing it from the stake.

The formulation of the problem is based on beam theory and its application to a growing structure (Archer 1986). For the sine model (similar to Fournier et al. 2006; Alméras and Fournier 2009), explicit formulae are provided to link maturation strain and curvature, in the free up-righting case (Eq. 27) and in the restrained case (Eq. 31). For the step-variation model, the basic equations enabling numerical computation of the model are provided. The reader not interested in mathematical developments may jump to the "Parametric study" section.

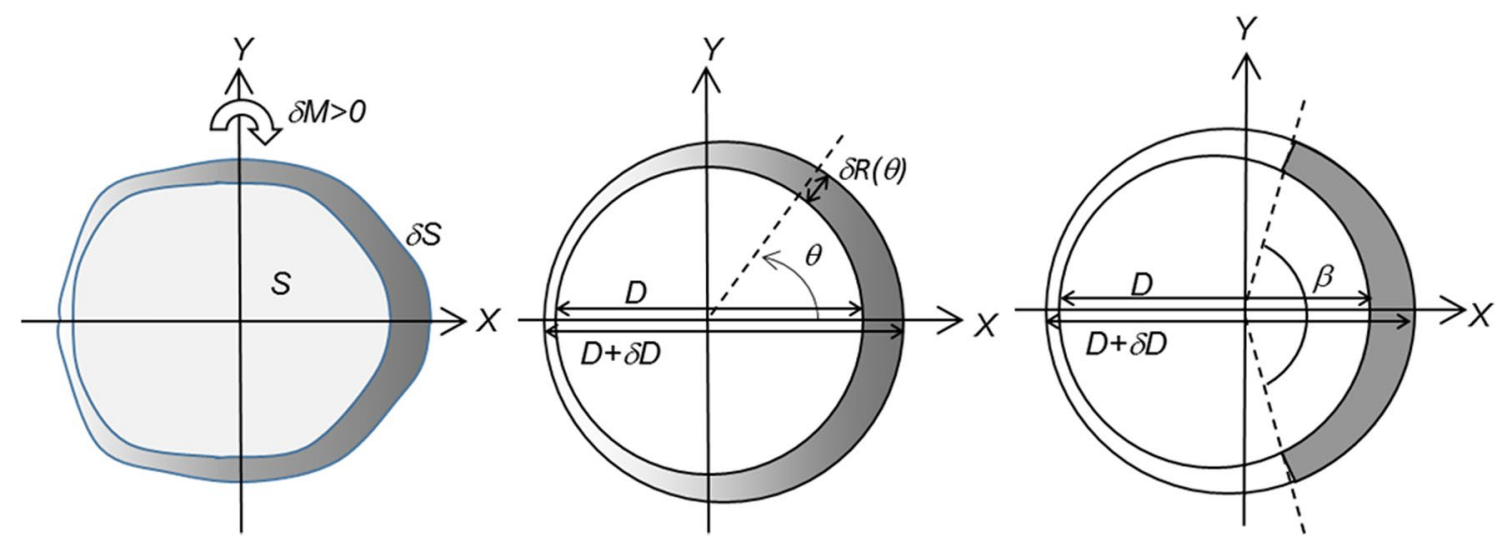

Fig. 1 Representations of a growing stem section. a Section of any shape with bilateral symmetry. The initial section is $S$ and the section increment is $\delta S$. Variation in grey level represents any variations in mechanical properties around the circumference of the section. Tissues with larger tension on the right side generate a bending moment $\delta M$ whose sign is indicated on the figure. $\mathbf{b}$ Representation with circular shape and sine variations in material properties. The initial section has diameter $D$ and the newly grown ring $D+\delta D$. The growth ring is supposed eccentric and the ring thickness at angle $\theta$ is $\delta R(\theta)$. Variation in grey level represents sine variations in mechanical properties around the circumference of the section. $\mathbf{c}$ Representation with circular shape, step variations in material properties and eccentric growth ring. Grey area represents the sector of reaction wood (here tension wood), with angular extension $\beta$ and specific mechanical properties 


\section{Representations of a growing stem section}

Three representations will be used to derive the model. The first (Fig. 1a) is suitable for the derivation of a general formulation, and later applied to parametric representations. The second representation (Fig. 1b) is based on that used by Alméras and Fournier (2009), with an eccentric growth ring with sinusoid variations of properties. These sine variations have interesting mathematical properties enabling the development of an explicit analytic formulation, but are not completely realistic to describe circumferential variations in maturation strain. The real situation is not antisymmetric between the side with reaction wood and the opposite side, particularly in the case of tension wood: reaction wood is generally located in a sector, and the circumferential variations in maturation strain are not as smooth as described with the sine model. The last representation (Fig. 1c) is, therefore, considering a sector of reaction wood, and will be used to derive a more accurate representation as described later. In present study, we illustrate the case of tension wood, but formulations remain correct when compression wood is considered.

\section{General formulation}

The formulation of the problem is based on beam theory. As the problem is assumed to have bilateral symmetry, only one component of the curvature (namely around Y, Fig. 1) is considered.

Let us consider a section growing between $t$ and $t+\delta t$ by adding a section increment $\delta S$. The section is submitted to internal loads increment due to impeded maturation strains $\delta \alpha$ distributed over the section, and external loads increment inducing a normal resultant force $\delta N_{\text {ext }}$ and a bending moment $\delta M_{\text {ext }}$. These loads generate strains $\delta \epsilon$ and stresses $\delta \sigma$ distributed within the section, resulting in an axial contraction $\delta \epsilon_{0}$ and a change in curvature $\delta C$ at the section level.

We consider that wood has elastic behaviour with prescribed strain, so that it follows Hooke's law:

$\delta \sigma=E(\delta \varepsilon-\delta \alpha)$

where $E$ is the elastic modulus of the material.

According to Bernoulli hypothesis underlying beam theory, the strain at a given position in the section is:

$\delta \varepsilon=\delta \varepsilon_{0}-x \delta C$,

where $x$ is the distance to the centre of the section and $\delta \varepsilon_{0}$ is the strain at the level of the centre of the section.

The condition for static equilibrium is:

$$
\begin{aligned}
& \delta N_{\mathrm{ext}}=\iint_{S} \delta \sigma \mathrm{d} s \\
& \delta M_{\mathrm{ext}}=-\iint_{S} x \delta \sigma \mathrm{d} s .
\end{aligned}
$$

Combining Eqs. (1) and (3) we obtain:

$$
\begin{aligned}
& \delta N_{\mathrm{ext}}=\iint_{S} E \delta \varepsilon \mathrm{d} s-\iint_{S} E \delta \alpha \mathrm{d} s \\
& \delta M_{\mathrm{ext}}=-\iint_{S} x E \delta \varepsilon \mathrm{d} s+\iint_{S} x E \delta \alpha \mathrm{d} s .
\end{aligned}
$$

We consider that the maturation strain is non-zero only on the newly produced wood ring, hence:

$\delta \alpha=0$ on $S$

$\delta \alpha=\alpha$ on $S$.

Combining Eqs. (4) and (5) we obtain:

$$
\begin{aligned}
& \delta N_{\mathrm{ext}}=\iint_{S} E \delta \varepsilon \mathrm{d} s-\iint_{\delta S} E \alpha \mathrm{d} s \\
& \delta M_{\mathrm{ext}}=-\iint_{S} x E \delta \varepsilon \mathrm{d} s+\iint_{\delta S} x E \alpha \mathrm{d} s .
\end{aligned}
$$

We define the axial force and bending moment induced by maturation as:

$$
\begin{aligned}
& \delta N_{\text {mat }}=\iint_{\delta S} E \alpha \mathrm{d} s \\
& \delta M_{\text {mat }}=-\iint_{\delta S} x E \alpha \mathrm{d} s .
\end{aligned}
$$

We define the terms of stiffness of the section as:

$$
\begin{aligned}
& K_{0}=\iint_{S} E \mathrm{~d} s \\
& K_{1}=-\iint_{S} x E \mathrm{~d} s \\
& K_{2}=\iint_{S} x^{2} E \mathrm{~d} s .
\end{aligned}
$$


Combining Eqs. (2), (6-8) and considering an infinitesimal growth increment $(\delta S \rightarrow \mathrm{d} S)$ we obtain:

$\frac{\mathrm{d} \varepsilon_{0}}{\mathrm{~d} S} K_{0}+\frac{\mathrm{d} C}{\mathrm{~d} S} K_{1}=\frac{\mathrm{d} N_{\text {ext }}}{\mathrm{d} S}+\frac{\mathrm{d} N_{\text {mat }}}{\mathrm{d} S}$

$\frac{\mathrm{d} \varepsilon_{0}}{\mathrm{~d} S} K_{1}+\frac{\mathrm{d} C}{\mathrm{~d} S} K_{2}=\frac{\mathrm{d} M_{\mathrm{ext}}}{\mathrm{d} S}+\frac{\mathrm{d} M_{\mathrm{mat}}}{\mathrm{d} S}$.

\section{Case of free up-righting}

In the case of free up-righting, the external loads are zero:

$\frac{\mathrm{d} N_{\text {ext }}}{\mathrm{d} S}=0$
$\frac{\mathrm{d} M_{\text {ext }}}{\mathrm{d} S}=0$.

The elementary variation in curvature can be deduced from (9) and (10):

$\frac{\mathrm{d} C}{\mathrm{~d} S}=\frac{K_{0} \mathrm{~d} M_{\text {mat }} / \mathrm{d} S-K_{1} \mathrm{~d} N_{\text {mat }} / \mathrm{d} S}{K_{0} K_{2}-K_{1}^{2}}$.

The total variation in curvature for a section growing from $S_{0}$ to $S_{1}$ is obtained by integration:

$\Delta C=\int_{S_{0}}^{S_{1}} \frac{\mathrm{d} C}{\mathrm{~d} S} \mathrm{~d} S$.

\section{Case of restrained stem}

In the restrained case, the external resultant force and the change in curvature are zero:

$\frac{\mathrm{d} N_{\text {ext }}}{\mathrm{d} S}=0$

$\frac{\mathrm{d} C}{\mathrm{~d} S}=0$.

The increment in external bending moment can be deduced from (9) and (13):

$\frac{\mathrm{d} M_{\mathrm{ext}}}{\mathrm{d} S}=\frac{K_{1}}{K_{0}} \frac{\mathrm{d} N_{\mathrm{mat}}}{\mathrm{d} S}-\frac{\mathrm{d} M_{\mathrm{mat}}(t)}{\mathrm{d} S}$.

The total variation in bending moment for a section growing from $S_{0}$ to $S_{1}$ can be obtained by integration:

$\Delta M_{\mathrm{ext}}=\int_{S_{0}}^{S_{1}} \frac{\mathrm{d} M_{\mathrm{ext}}}{\mathrm{d} S} \mathrm{~d} S$.

The final change in curvature when releasing the stem from the stake is:

$\Delta C=-\Delta M_{\mathrm{ext}} \frac{K_{0}\left(S_{1}\right)}{K_{0}\left(S_{1}\right) K_{2}\left(S_{1}\right)-K_{1}^{2}\left(S_{1}\right)}$.
Note that this model, although general, neglects the effect of bark, whose stiffness and thickness are generally low.

\section{Simplified analytical model with sine variations of material properties}

The first model is based on the representation of a stem section similar to Alméras and Fournier (2009).

The section is assumed circular, with sine variations of material properties:

$\alpha(\theta)=\bar{\alpha}+\frac{\Delta \alpha}{2} \cos (\theta)=\bar{\alpha}\left(1+k_{\alpha} \cos \theta\right)$,

$E(\theta)=\bar{E}+\frac{\Delta E}{2} \cos (\theta)=\bar{E}\left(1+k_{\mathrm{E}} \cos \theta\right)$.

With:

$k_{\alpha}=\frac{\Delta \alpha}{2 \bar{\alpha}}=\frac{\alpha(0)-\alpha(\pi)}{\alpha(0)+\alpha(\pi)}$,

$k_{\mathrm{E}}=\frac{\Delta E}{2 \bar{E}}=\frac{E(0)-E(\pi)}{E(0)+E(\pi)}$.

Variations in ring thickness due to eccentric growth are defined by:

$\delta R(\theta)=\delta \bar{R}\left(1+k_{O} \cos \theta\right)$.

With:

$k_{O}=\frac{\delta R(0)-\delta R(\pi)}{\delta R(0)+\delta R(\pi)}$.

Combining Eqs. (7), (17-19), the bending moment induced by maturation can be computed as:

$$
\begin{aligned}
\delta M_{\text {mat }}=-\delta \bar{R} \bar{E} \bar{\alpha} \int_{0}^{2 \pi} R \cos \theta\left(1+k_{\mathrm{O}} \cos \theta\right) \\
\left(1+k_{\mathrm{E}} \cos \theta\right)\left(1+k_{\alpha} \cos \theta\right) R \mathrm{~d} \theta \\
=-\pi R^{2} \delta \bar{R} \bar{E} \bar{\alpha}\left(k_{\alpha}+k_{\mathrm{E}}+k_{\mathrm{O}}+\frac{3}{4} k_{\alpha} k_{\mathrm{E}} k_{\mathrm{O}}\right) .
\end{aligned}
$$

(integrals of cosine power functions are provided in Appendix).

\section{Case of free up-righting}

Here, we assume that the neutral line is at the geometric centre of the section. Note that this assumption is not consistent with the assumption of heterogeneous elastic modulus, and is potentially a source of error. Making this approximation, $K_{1}=0$, Eq. (11) reduces to:

$\delta C=\frac{\delta M_{\mathrm{mat}}}{K_{2}}$. 
For a circular cross section with mean modulus of elasticity $\bar{E}$, the bending stiffness is given by:

$K_{2}=\bar{E} \frac{\pi R^{4}}{4}$.

Combining Eqs. (20-22), the curvature increment is:

$\delta C=-4 \bar{\alpha} \frac{\delta \bar{R}}{R^{2}}\left(k_{\alpha}+k_{\mathrm{E}}+k_{\mathrm{O}}+\frac{3}{4} k_{\alpha} k_{\mathrm{E}} k_{\mathrm{O}}\right)$.

Considering that $D=2 R$ and $\delta D=2 \delta \bar{R}$, this can be put in the same form as in Alméras and Fournier (2009):

$\delta C=-4 \Delta \alpha f \frac{\delta D}{D^{2}}$

With the form factor $f$ defined as:

$f=1+\left(k_{\mathrm{E}}+k_{\mathrm{O}}\right) / k_{\alpha}+\frac{3}{4} k_{\mathrm{E}} k_{\mathrm{O}}$.

From Eq. (24) and considering an infinitesimal diameter increment $(\delta D \rightarrow \mathrm{d} D)$ it comes that:

$\frac{\mathrm{d} C}{\mathrm{~d} D}=-\frac{4 \Delta \alpha f}{D^{2}}$

The total variation in curvature is obtained after integration:

$\Delta C=\int_{D_{0}}^{D_{1}} \frac{\mathrm{d} C}{\mathrm{~d} D} \mathrm{~d} D=4 \Delta \alpha f\left(\frac{1}{D_{1}}-\frac{1}{D_{0}}\right)$.

\section{Case of restrained stem}

Assuming the neutral line is at the geometric centre of the section, $K_{1}(t)=0$, Eq. (14) reduces to:

$d M_{\mathrm{ext}}=-d M_{\mathrm{mat}}$

Considering that $D=2 R$ and $\delta D=2 \delta \bar{R}$ and rearranging (20), we obtain:

$\frac{\mathrm{d} M_{\mathrm{mat}}}{\mathrm{d} D}=-\frac{\pi}{16} D^{2} \bar{E} \Delta \alpha f$.

Applying Eq. (15), we obtain:

$\Delta M_{\text {ext }}=-\int_{D_{0}}^{D_{1}} \mathrm{~d} M_{\text {mat }}=-\frac{\pi}{16} \bar{E} \Delta \alpha f \frac{D_{1}^{3}-D_{0}^{3}}{3}$.

Considering (9) and (22), the variation in curvature when releasing the stem from the stake is:

$\Delta C=\frac{\Delta M_{\mathrm{ext}}}{K_{2}\left(t_{1}\right)}=-\frac{4}{3} \Delta \alpha f \frac{D_{1}^{3}-D_{0}^{3}}{D_{1}^{4}}$.

\section{Model with step distribution of material properties}

Reaction wood is here assumed to be located in a sector of the ring, given by angular extension $\beta$. The remaining of the section is supposed to be made of normal wood. The distribution of material properties $E$ and $\alpha$ is assumed uniform over each sector.

The maturation loads are then given as:

$$
\begin{aligned}
\delta N_{\mathrm{mat}} & =\iint_{\delta S} E \alpha \mathrm{d} s \\
& =\alpha_{\mathrm{RW}} \mathrm{E}_{\mathrm{RW}} \iint_{\delta S_{\mathrm{RW}}} \mathrm{d} s+\alpha_{\mathrm{NW}} E_{\mathrm{NW}} \iint_{\delta S_{\mathrm{NW}}} \mathrm{d} s \\
& =\alpha_{\mathrm{RW}} E_{\mathrm{RW}} \delta S_{\mathrm{RW}}+\alpha_{\mathrm{NW}} E_{\mathrm{NW}} \delta S_{\mathrm{NW}} \\
\delta M_{\mathrm{mat}} & =-\iint_{\delta S} x E \alpha \mathrm{d} s \\
& =-\alpha_{\mathrm{RW}} E_{\mathrm{RW}} \iint_{\delta S_{\mathrm{RW}}} x \mathrm{~d} s-\alpha_{\mathrm{NW}} E_{\mathrm{NW}} \iint_{\delta S_{\mathrm{NW}}} x \mathrm{~d} s \\
& =-\alpha_{\mathrm{RW}} E_{\mathrm{RW}} \delta J_{\mathrm{RW}}-\alpha_{\mathrm{NW}} E_{\mathrm{NW}} \delta J .
\end{aligned}
$$

$S$ and $J$ are the area and the first moment of area, with subscript RW for reaction wood and NW for normal wood, and $\delta$ indicates their increment. Let $I$ be the second moment of area. For each radius increment $\delta R$, the increment in area and moments of area relative to the centre of the ring of an eccentric sector limited by angle $\theta_{1}$ and $\theta_{2}$ are:

$$
\begin{aligned}
\delta S_{\theta_{1}, \theta_{2}}^{*} & =R \delta \bar{R} \int_{\theta_{1}}^{\theta_{2}}\left(1+k_{\mathrm{O}} \cos \theta\right) \mathrm{d} \theta \\
& =R \delta R\left(\phi_{0}\left(\theta_{1}, \theta_{2}\right)+k_{O} \phi_{1}\left(\theta_{1}, \theta_{2}\right)\right) \\
\delta J_{\theta_{1}, \theta_{2}}^{*} & =R^{2} \delta \bar{R} \int_{\theta_{1}}^{\theta_{2}} \cos \theta\left(1+k_{\mathrm{O}} \cos \theta\right) \mathrm{d} \theta \\
& =R^{2} \delta R\left(\phi_{1}\left(\theta_{1}, \theta_{2}\right)+k_{O} \phi_{2}\left(\theta_{1}, \theta_{2}\right)\right) \\
\delta I_{\theta_{1}, \theta_{2}}^{*} & =R^{3} \delta \bar{R} \int_{\theta_{1}}^{\theta_{2}} \cos ^{2} \theta\left(1+k_{\mathrm{O}} \cos (\theta)\right) \mathrm{d} \theta \\
& =R^{3} \delta R\left(\phi_{2}\left(\theta_{1}, \theta_{2}\right)+k_{O} \phi_{3}\left(\theta_{1}, \theta_{2}\right)\right),
\end{aligned}
$$

where $\phi_{0}, \phi_{1}, \phi_{2}$ and $\phi_{3}$ are integrals of cosine powers provided in Appendix.

Area and moments of area for the computation of loads and stiffness must be computed relative to a fixed reference. For an eccentric case, the centre of the section is not fixed. The fixed reference is taken at the pith, i.e. the centre of the initial section of radius $R_{0}$. The position of the centre of a ring with radius $R$ relative to the pith is given by:

$x_{0}=k_{O}\left(R-R_{0}\right)$. 
Increments in moments of area computed at the pith $\delta S, \delta J$, $\delta I$ can be deduced from those computed at the centre of the section $\delta S^{*}, \delta J^{*}, \delta I^{*}$ :

$\delta S=\delta S^{*}$

$\delta J=\delta J^{*}+x_{0} \delta S^{*}$

$\delta I=\delta I^{*}+x_{0}^{2} \delta S^{*}$.

Moment of area for reaction and normal wood sectors can be computed from these equations. The loads (normal force and bending moment) can be deduce using (31).

Stiffness increments for an increment in section are given by:

$\delta K_{0}=\iint_{\delta S} E \mathrm{~d} s=E_{\mathrm{RW}} \delta S_{\mathrm{RW}}+E_{\mathrm{NW}} \delta S_{\mathrm{NW}}$

$\delta K_{1}=\iint_{\delta S} x E \mathrm{~d} s=E_{R W} \delta J_{R W}+E_{N W} \delta J_{\mathrm{NW}}$

$\delta K_{2}=\iint_{\delta S} x^{2} E \mathrm{~d} s=E_{\mathrm{RW}} \delta I_{\mathrm{RW}}+E_{\mathrm{NW}} \delta I_{\mathrm{NW}}$.

Total stiffness terms $K_{0}, K_{1}, K_{2}$ can be computed by integration. Any radial pattern of variations in modulus of elasticity and eccentricity can be considered with this formulation. In particular, the presence of a pith with finite diameter and negligible stiffness can easily be taken into account.

The variations in curvature in the free up-righting and restrained cases can be deduced from Eqs. (11-12) and (15, 16), respectively.

\section{Model implementation and inversion}

Above described models enable the calculation of the variation in curvature $\Delta C$ as a function of section's parameters, and in particular the value the maturation strain of reaction wood. For the sine model, the solution can be obtained analytically from Eqs. (27) and (31). The step-variation model has to be computed numerically. It was implemented using Microsoft Excel and Visual Basic. The estimation of reaction wood maturation strains $\alpha_{\mathrm{RW}}$ can be obtained by inversion of the model. As the dependence of $\Delta C$ in $\alpha_{\mathrm{RW}}$ is linear, this inversion is trivial.

\section{Parametric study: influence of section parameters on the magnitude of reaction}

This parametric study aims on one hand at analysing the influence of the section parameters on the performance of the reaction, namely the variation in curvature, and on the other hand at comparing the result of the analytical sine
Table 1 Range of values used for the parametric study. TW: tension wood, NW: normal wood, MOE: modulus of elasticity

\begin{tabular}{llll}
\hline & Unit & Reference value & Range \\
\hline Initial diameter & $\mathrm{mm}$ & 5 & $1-10$ \\
$\begin{array}{l}\text { Relative diameter } \\
\text { increment }\end{array}$ & - & 0.5 & $0-3$ \\
Pith relative radius & - & 0.4 & $0-0.95$ \\
TW extension & $\circ$ & 135 & $0-180$ \\
Eccentricity & - & 0.67 & $0-0.95$ \\
NW MOE & MPa & 2800 & $1000-10,000$ \\
TW/NW MOE & - & 1.2 & $0.5-2$ \\
Maturation strain TW & $\mu$ strain & -5000 & -500 to $-10,000$ \\
\hline
\end{tabular}

model and the numerical step model. This analysis will be conducted on the two cases considered, namely free uprighting and restrained stem. The principle of this analysis is that the response to each parameter is computed for fixed value of other parameters. The section parameters are illustrated in Fig. 4. The reference values used for the study (Table 1) are close to the mean value measured in the experimental study described later. For each parameter, the range of variation represents a reasonable range of biologically meaningful values for broad-leaf trees, i.e. trees with tension wood.

\section{Analysis of the influence of sections parameters}

Results are shown on Fig. 2. In this section, only the results of the model with step variations in properties (solid lines) will be commented. The comparison with the sine model (dashed lines) will be commented in next section.

Results show that in all cases the variation in curvature is approximately twice lower in the restrained case compared to the free up-righting case. This can be explained considering the difference between the two cases. In the restrained case, the bending moment accumulated during growth is applied on the final structure, with large stiffness. In contrast, in the case of free up-righting, each moment increment is applied to the structure at the time it appears, so that early increment are applied on a structure with lower stiffness, inducing a larger change in curvature.

Figure 2-a shows that the variation in curvature has strong inverse response to the section diameter, as could be predicted from Eqs. (27) and (31). Figure 2-b shows that for the free up-righting case the reaction increases with the relative radius increment, due to the fact that variations in curvature are cumulated during growth. By contrast, in the restrained case, the response presents optimum for a relative increment close to $50 \%$. The occurrence of this optimum can be deduced from Eq. (31). This has consequences on the practical application of this model, i.e. experimental 

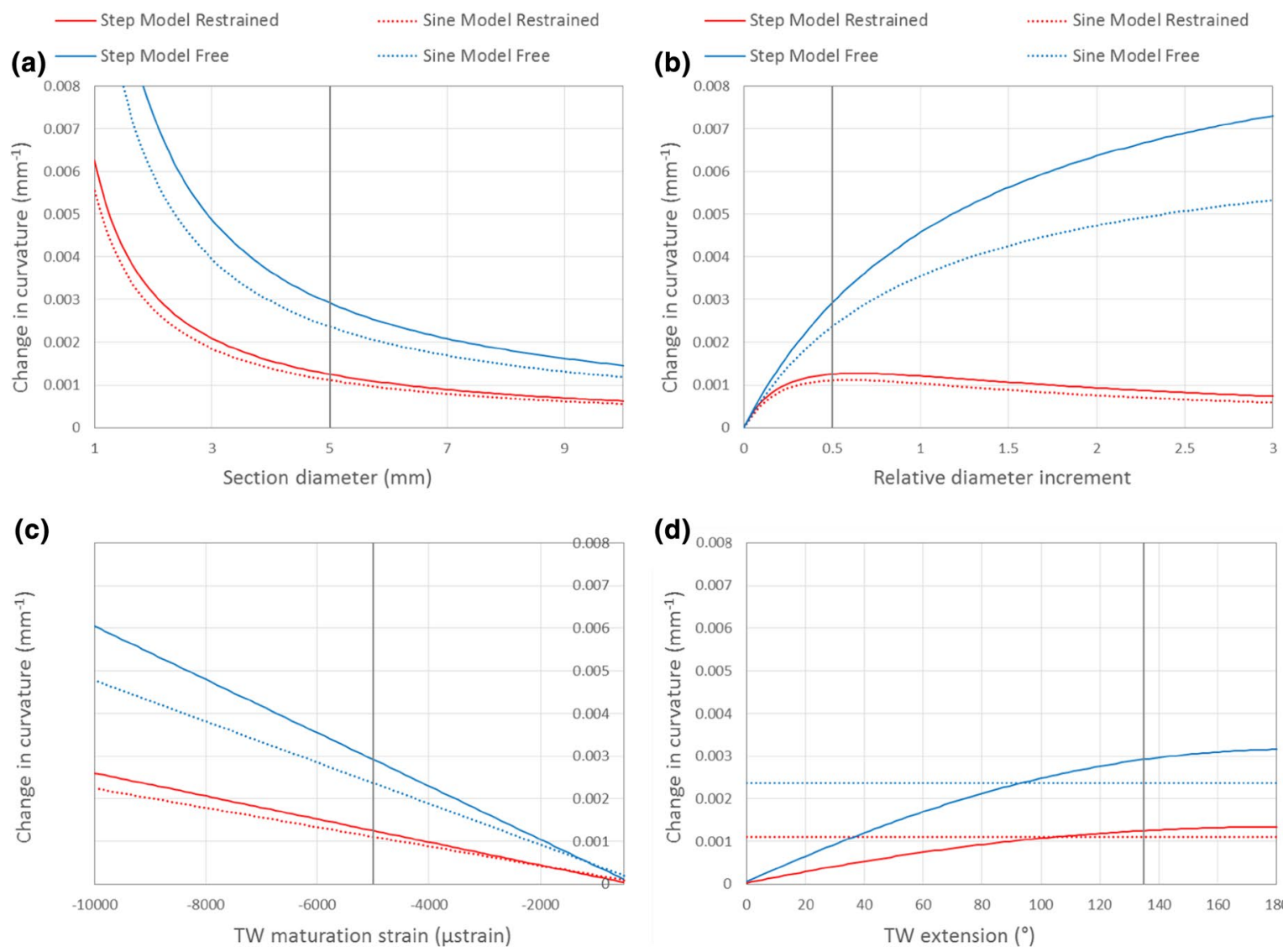

(e)
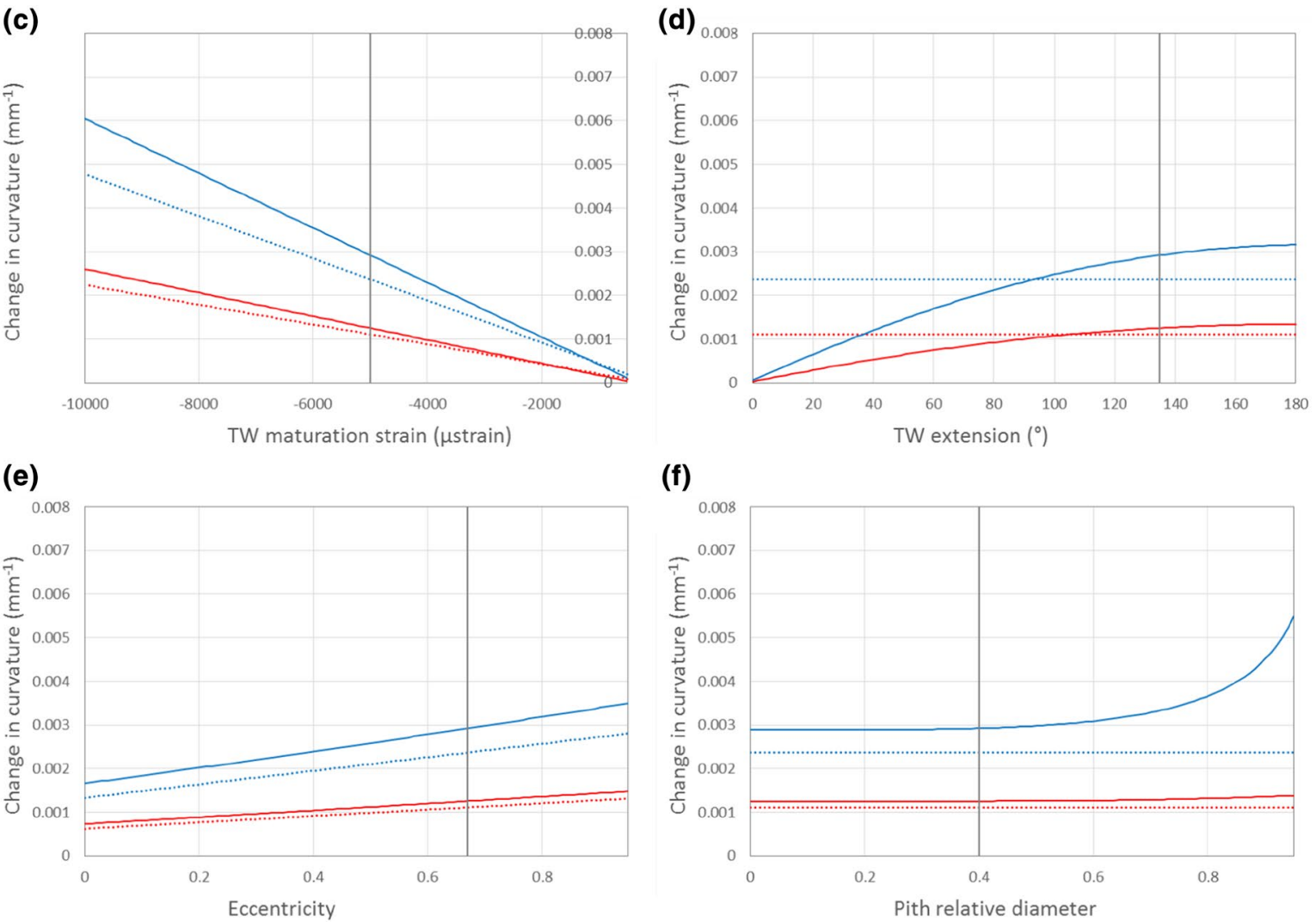

(g)

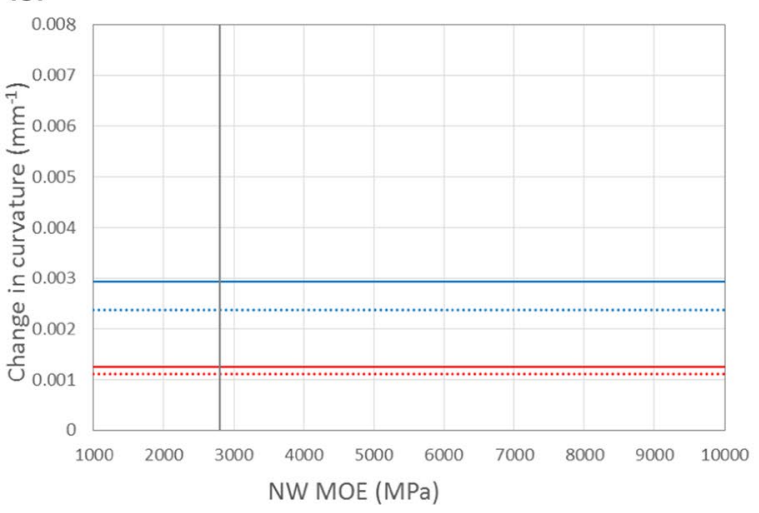

(f)

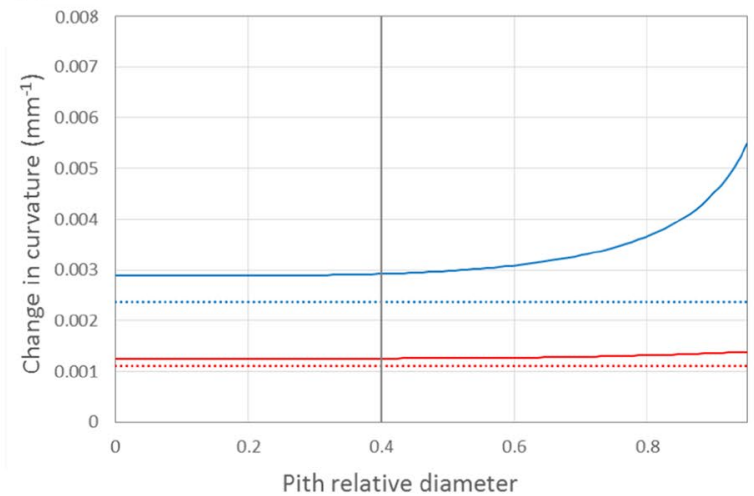

(h)

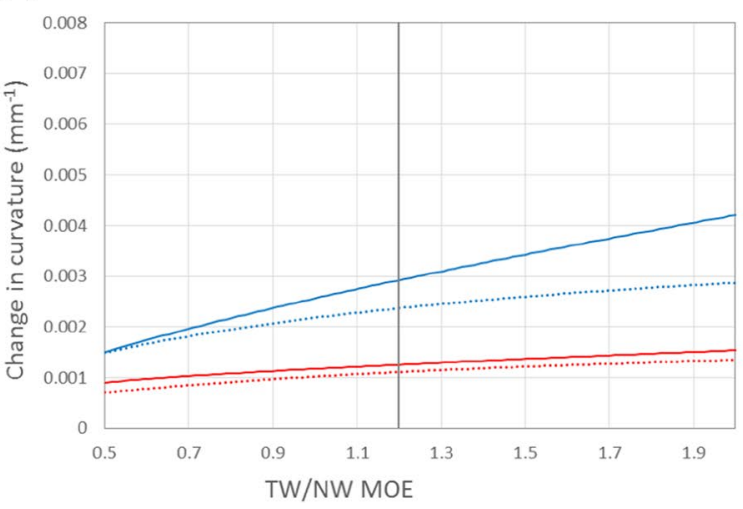


4Fig. 2 Results of the parametric study. Results are given for the two representations (step model and sine model) and for the two cases (free up-righting and restrained reaction). Vertical lines indicates the reference value used when varying other parameters

determination of tension wood maturation strain. Obtaining a maximal reaction by setting a $50 \%$ diameter increment improves the accuracy of this measurement as it decreases the relative error on the estimation of curvature.

Figure $2 \mathrm{c}$ shows that the response of the curvature is linear in the value of tension wood maturation strain for both cases, as predicted from the equations. This strong response shows that, as previously stated (Fournier et al. 2006), tension wood maturation strain acts as a predominant effect, being the motor of the reaction. The extension of tension wood (Fig. 2d) is also a predominant effect, although it has concave shape in both cases, so that the response is less sensitive for large values of tension wood extension (typically values larger than usually observed).

Other parameters are second-order effects, with less sensitive variations and non-zero values of change in curvature for the minimal values set for the parameter. Effect of eccentricity (Fig. 2e) is linear, and that of the MOE ratio (Fig. 2h) almost linear. In the restrained case, it can be noticed that the effect of MOE ratio is weak, so that small variations in this parameter can be neglected. For the free up-right model, pith dimension may have a large effect if it has large values (Fig. 2f), typically in the case where the stem is very young. This is due to the reduction in stiffness of the stem when pith is large, so that early increments in bending moment act on a compliant structure yielding large changes in curvature. In contrast, pith always has a negligible effect in the restrained case, because its contribution to the stem stiffness after growth is always negligible, due to the dependence between second moment of area and diameter at power 4 . Finally, normal wood MOE (for a given TW/NW MOE ratio, Fig. $2 \mathrm{~g}$ ) has no effect on the performance of the reaction, because both the bending moment and the stiffness are proportional to it. Only the MOE ratio (Fig. 2h) plays a role, by increasing the bending moment for a given maturation strain.

\section{Comparison between the sine and step-variation models}

The step-variation model will be taken as a reference, because it is more accurate (by taking into account the changes in neutral line when MOE is heterogeneous) and closer to reality (as variations in maturation strain are closer to a step variation than a sine variation).

Figure 2 shows that the sine model always underestimates the curvature compared to the step-variation model. This underestimation of approximately $-10 \%$ for the restrained case and $-20 \%$ for the free up-righting case implies an equivalent overestimation when the reverse model is used to estimate the maturation strain of tension wood. The sine model as derived does not reflect the effects of the pith (Fig. 2f) and tension wood extension (Fig. 2d). For other parameters, the trend of the curve is the same as for the step-variation model.

Further parametric analyses (not shown) show that the results of the sine model are exactly equivalent to the stepvariation model when there is no variations in MOE and a tension wood sector of $103^{\circ}$ is considered. This critical value actually depends on parameters such as MOE ratio and eccentricity. As stated by Alméras ad Fournier (2009), this equivalence is explained by the fact that the sine model can be considered as the first-order development of a Fourier series describing any variation in properties, for which higher order terms vanish when integrated.

\section{Experimental validation of the method}

The method was applied on two different datasets. Results presented in this section aim at validating the method by demonstrating its capacity to discriminate between different plant genotypes and to suppress first-order dimensional effects. The biological interpretation of the results will not be commented further as it is not the focus of this paper.

\section{Plant material}

The first study aimed at studying the diversity of the motor function among tropical species. It was conducted on 132 young trees from 16 tropical species. Trees were grown in a greenhouse from seeds or seedlings collected in the forest. The species chosen represent a wide diversity of morphology, their diameter at the end of the experiment ranging from 4 to $16 \mathrm{~mm}$. After being acclimated in a greenhouse, stems were tied to a stake and tilted at an angle of $45^{\circ}$ from vertical. Thereafter, trees were grown in tilted position until their external diameter reached approximately 1.5 times the initial diameter to maximize the change in curvature (see section "parametric study"). They reached this diameter after variable time, due to the diversity of growth rates between species. Finally, plants were collected and measured as described in next section.

The second study aimed at unravelling the mechanism of maturation strain generation in tension wood. It was conducted on three different poplar transgenic lines modified for a gene potentially involved in tension wood formation, as well as the wild-type untransformed line, the hybrid Populus tremula $x$ Populus alba clone INRA 717-1B4. Three replicate trees per line were grown from microcuttings in a greenhouse during 4.5 months, until they reached a height of $1.2 \mathrm{~m}$, with little variations between trees. Then they were 
Fig. 3 Illustration of the tree reaction. Left: stem tied to a stake that has grown tilted. Right: spring-back movement after releasing the stem from the stake
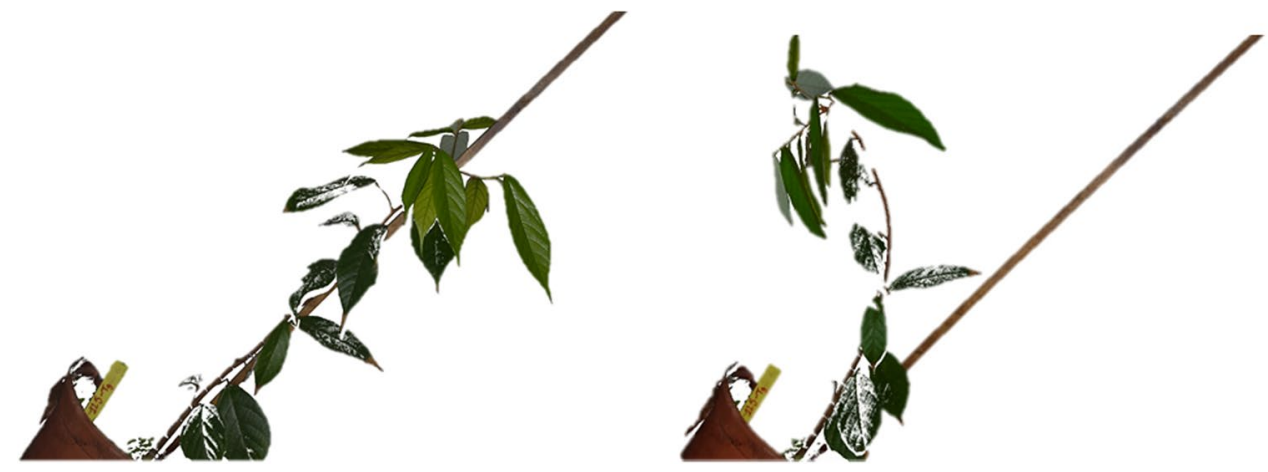

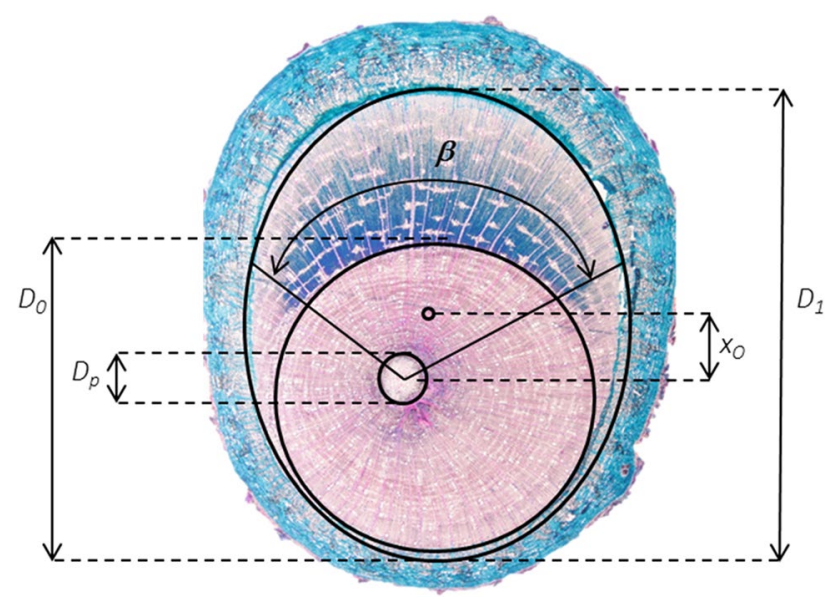

Fig. 4 Parameter describing section's morphology. $D_{0}$ : Initial wood diameter; $D_{1}$ : final wood diameter; $D_{\mathrm{p}}$ : diameter of the pith; $x_{\mathrm{O}}$ : position of the centre of the final wood section relative to the pith; $\beta$ : extension of the tension wood sector

tied to a stake and tilted at $35^{\circ}$ degrees from vertical, grown during 10 weeks in this position and finally collected and measured as described below.

\section{Measurements}

The measurement protocol was similar for both studies. Trees were released from the stake, generating an instantaneous spring-back movement, so that the stem was curved, and sometimes passed the vertical (Fig. 3). A segment of the stem located at its middle was then cut and placed in horizontal position on the floor, to supress the effect of self-weight. A picture of the segment was taken to quantify the change in curvature due to stem reaction. Curvature was measured by locating points along the segment using an image analysis software, and fitting them to a polynomial function, from which curvature was derived analytically.

In both studies, a thin section was cut from the middle of the segment to analyse the morphology of the section. All sections showed a sector of tension wood located on the upper part of the section (Fig. 4), with more or less pronounced eccentric growth depending on species. Different parameters were measured to describe the section: diameter of the initial section $D_{0}$ (before tilting), final dimension of the section $D_{1}$, diameter of the pith, location of the pith centre (to quantify the eccentricity parameter $k_{\mathrm{O}}$ ) and extension of the tension wood sector $\beta$ (Fig. 4). As the maturation strain of normal wood $\alpha_{N W}$ is low and has low variability, a value $-500 \mu$ strain was assumed for this parameter, close to experimentally measured values (Clair et al. 2006, 2013, Fournier 1994). Other parameters were set at their measured value.
Table 2 Analyses of variance of the effect of genotype on tension wood maturation strain

\begin{tabular}{|c|c|c|c|c|c|c|}
\hline Source & DOF & Sum Sq & & Mean Sq & $F$ & $\operatorname{Pr}>F$ \\
\hline \multicolumn{7}{|c|}{ First study (Poplar transgenic lines) } \\
\hline Model & 4 & $7.60 \mathrm{E}-06$ & & $1.90 \mathrm{E}-06$ & 5.330 & 0.001 \\
\hline Error & 46 & $1.64 \mathrm{E}-05$ & & $3.56 \mathrm{E}-07$ & & \\
\hline Total & 50 & $2.40 \mathrm{E}-05$ & & & & \\
\hline \multicolumn{7}{|c|}{ Second study (Tropical diversity) } \\
\hline Model & 15 & & $5.51 \mathrm{E}-04$ & $3.67 \mathrm{E}-05$ & 10.951 & $<0.0001$ \\
\hline Error & 116 & & $3.89 \mathrm{E}-04$ & $3.36 \mathrm{E}-06$ & & \\
\hline Total & 131 & & $9.40 \mathrm{E}-04$ & & & \\
\hline
\end{tabular}


(a)

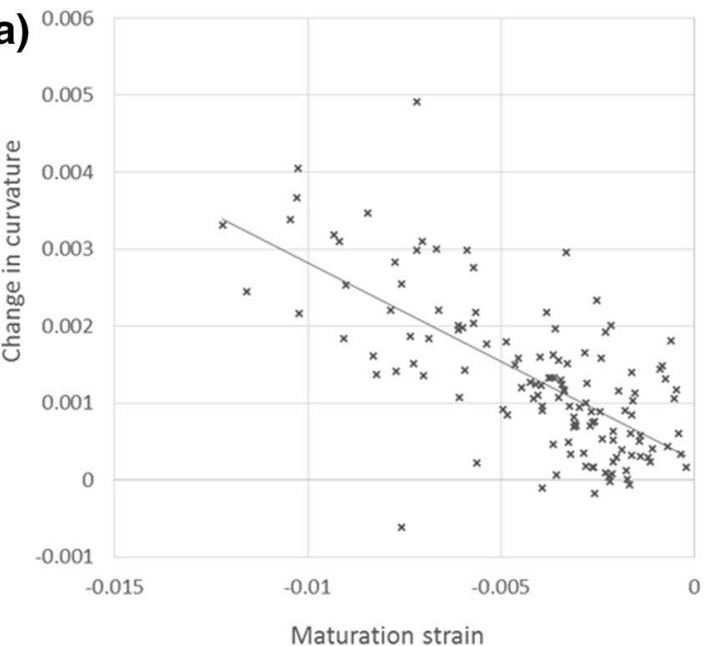

(b)

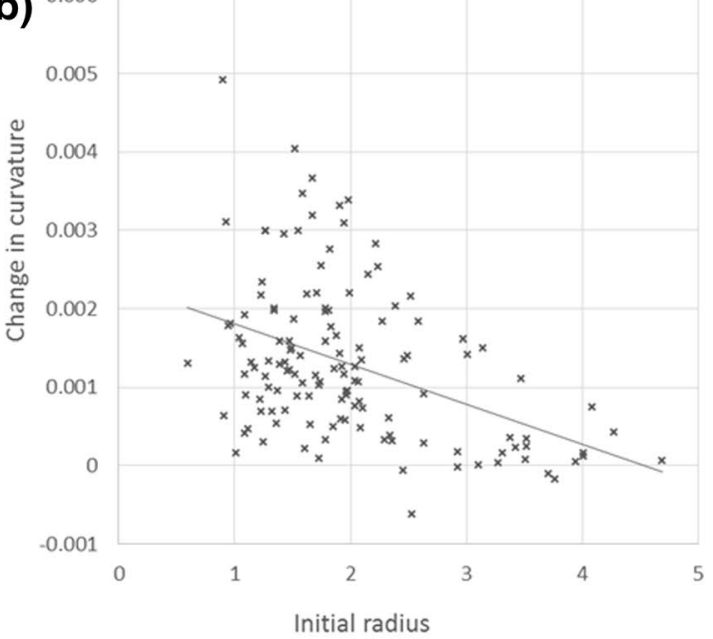

(d)



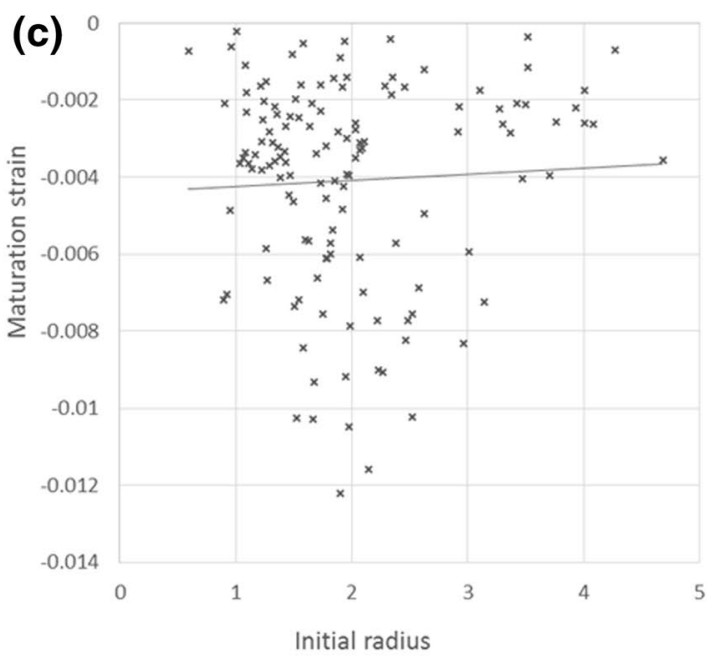

(e)

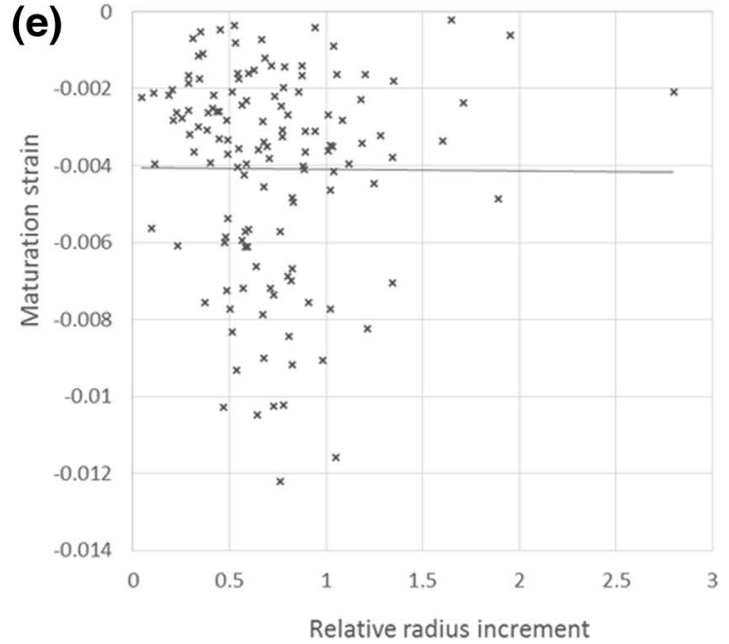

Fig. 5 Suppression of dimensional effects by the model: the change in curvature is strongly correlated to initial diameter and slightly correlated with relative growth increment. Computation of tension wood maturation strain suppresses these correlations 


\section{The model is able to discriminate between genotypes}

The model with step variation of material properties was used to compute the maturation strain of tension wood $\left(\alpha_{\mathrm{TW}}\right)$ from the change in curvature $\Delta C$. Estimated maturation strains ranged between -1300 and $-7200 \mu$ strains, with variations between specimens and between species. Oneway analysis of variance evidences significant differences between species for the first study $(P<0.001$, Table 2$)$, and between transgenic lines for the second study $(P<0.001$, Table 2). This demonstrates the discriminating power of the method and, therefore, its suitability to analyse variations in tension wood maturation strain intensity for various research purposes.

\section{Scaling effects are suppressed by the model}

Figure 5a shows the relation between the change in curvature $\Delta C$ and computed maturation strain $\alpha_{\mathrm{TW}}$. These parameters are correlated $\left(R^{2}=0.48, P<0.0001\right)$ because, as mentioned earlier, the maturation strain has a predominant effect on the change in curvature. However, more than $50 \%$ of the variance of the change in curvature is not explained by the maturation strain. Figure 5-b shows that change in curvature is also dependant on the initial radius of the stem $R_{0}$ $\left(R^{2}=0.18, P<0.0001\right)$. This is directly related to the size effect on curvature previously mentioned. In contrast, the maturation strain is not correlated to stem diameter (Fig. 5c, $R^{2}=0.002, P>0.05$ ). This demonstrates that the analysis of curvature is not appropriate for estimating the magnitude of the reaction at the material level, because it includes effects of section dimensions that are not directly related to material properties: the smaller the section, the larger the change in curvature, irrespective of the magnitude of maturation strain.

Figure 5-d shows that the change in curvature is practically uncorrelated $\left(R^{2}=0.013, P>0.05\right)$ to the relative radius increment. This is consistent with the weak dependence shown in Fig. 2b. The correlation between maturation strain and relative radius increment (Fig. 5e) is even lower $\left(R^{2}<0.001, P>0.05\right)$, showing that the model captures this weak effect size effect.

\section{Discussion and conclusion}

\section{Magnitude of estimated maturation strains}

The range of maturation strain values encompass values usually measured with the strain-release method on adult trees (Clair et al. 2006, 2013; Fournier et al. 1994), but values larger than usual (namely larger than $~ 4000 \mu$ strains) are also found. At least two reasons justify that measured values are larger than those measured with the strain-release method on adult trees. First, the strain-release method intrinsically tends to underestimate the magnitude of real maturation strains as argued in introduction. Second, measurements performed in the current study are made on severely tilted and restrained stems, so that the expression of the reaction is strong, and likely larger than in naturally tilted adult trees.

Values measured here are globally two times lower than the values of efficiency estimated with the free up-righting method in Alméras et al. (2009). This is related to the fact that in instance the free up-righting method was used to estimate a global efficiency at the section level, including the effect of form parameters (Eq. 25, Alméras and Fournier 2009; Alméras et al. 2009) rather than the value of maturation strain itself. As form parameter act in synergy with maturation strain, the efficiency at the section level, as described in Alméras et al. (2009), is intrinsically larger than the maturation strains itself.

\section{Estimating efficiency vs. estimating maturation strains}

The sine model reflects the effects of variations in MOE and eccentric growth, but does not account for tension wood extension. As a consequence, the value of $\Delta \alpha$ estimated by the sine model is not exactly equal to the difference in maturation strains between the two sides of the stem. It is the value that $\Delta \alpha$ would have if the variations were sine variations, and it is equivalent to the step-variation model only if for a particular value of the extension of tension wood. If the extension is larger, as usually the case, $\Delta \alpha$ estimated by the sine model overestimates the real difference in maturation strain.

The sine model is not able to directly estimate tension wood maturation strain, but is suitable to estimate an efficiency of the reaction as defined in Alméras et al. (2009), i.e. $\Delta \alpha f$ in Eqs. (27) and (31). The efficiency includes both the magnitude of the difference in maturation strain and the effect of form parameters such as eccentricity, distribution of MOE and real extension of tension wood. The same efficiency can be achieved, for example, either by a tension wood with large maturation strain or by a tension wood combining moderate maturation strain with eccentricity and variations in MOE. Compared to the measurement of curvature, it supresses first-order dimensional effects. It quantifies the intensity of the reaction for a given size (diameter) and a given cost (growth). It is suitable e.g. to compare globally the intensity of the reaction between plants, for example to study trade-offs between the motor function and other functions in an ecological context (Alméras et al. 2009), irrespective of the way a given efficiency is achieved.

The step-variation model enables the direct estimation of tension wood maturation strains. This parameter is suitable 
to compare biomechanical properties of different kinds of woods, for example belonging to different genotypes. Typically, this can be used to estimate the intensity of the tension wood produced in kinematic studies of gravitropism (Coutand et al. 2007; Alméras et al. 2009; Bastien et al. 2013), compare the motor power of different types of tension wood (Ruelle et al. 2007) or to study the mechanism generating the mechanical stress during tension wood formation (reviewed in Alméras and Clair 2016).

\section{The restrained stem method vs. the free up-righting method}

The restrained stem method has many advantages on the free up-righting method. The free up-righting movement of a stem is the result of two phenomena: the gravitropic up-righting and the autotropic straightening (Coutand et al. 2007; Bastien et al. 2013). The former is expressed in early stages of the uprighting process, and implies the production of tension wood in the upper side of the stem. The latter occurs in a second phase, and implies an inversion of stem curvature necessary to achieve a straight and vertical shape without overshooting the vertical. The stimulus (inclination) and the reaction are, therefore, not constant in time (during growth), neither in space (along the stem). This implies that the model can only be applied over short periods of time, and only on parts of the stem that are actively reacting. Thus, changes in curvature have to be repeatedly measured, and the model has to be applied on short growth increments. In contrast, during the growth of a restrained stem, the stimulus and reaction are kept constant in space and time, so that this method can be applied over the whole growth period. Less measurements are thus necessary and the method is more accurate.

Moreover, because the method is applied to the full period of growth, the morphological and physical properties of the section (initial diameter without bark, growth increment, eccentricity, tension wood extension, variations in MOE) obtained after stem sectioning can be used to feed the model. Furthermore, in the free up-righting method, the change in self-weight during up-righting has to be accounted for (Alméras et al. 2009), whereas in the case of the restrained stem it can be neglected. Finally, the effect of maturation kinetics (Coutand et al. 2007), i.e. the delay between tension wood formation and induction of maturation strains may have consequences in the case of the free up-righting method, whereas these consequences vanish when a large period of time is considered, as for the restrained stem method. The restrained stem method need less measurements and is more precise than the free-up-righting method to estimate the efficiency in the motor function. Application of this method to various research contexts should enable significant progress on the understanding of the motor function of trees.
Author contributions statement TA developed the model and wrote the paper. MF had the original idea and developed the model. BC and BG made the measurement for the study on tension wood tropical diversity. GP and AS made the measurements for the study on poplar transgenic lines.

Acknowledgements This work was done in the framework of the project 'StressInTrees' funded by the French National Research Agency (ANR-12-BS09-0004).

\section{Compliance with ethical standards}

Conflict of interest The authors declare that they have no conflict of interest.

\section{Appendix}

Integrals of cosine powers:

$\phi_{0}\left(\theta_{1}, \theta_{2}\right)=\int_{\theta_{1}}^{\theta_{2}} \mathrm{~d} \theta=\theta_{2}-\theta_{1}$,

$\phi_{1}\left(\theta_{1}, \theta_{2}\right)=\int_{\theta_{1}}^{\theta_{2}} \cos \theta \mathrm{d} \theta=[-\sin \theta]_{\theta_{1}}^{\theta_{2}}$

$\phi_{2}\left(\theta_{1}, \theta_{2}\right)=\int_{\theta_{1}}^{\theta_{2}} \cos ^{2} \theta \mathrm{d} \theta=\left[\frac{2 \theta+\pi+\sin 2 \theta}{4}\right]_{\theta_{1}}^{\theta_{2}}$,

$\phi_{3}\left(\theta_{1}, \theta_{2}\right)=\int_{\theta_{1}}^{\theta_{2}} \cos ^{3} \theta \mathrm{d} \theta=\left[\frac{-\sin \theta\left(2+\cos ^{2} \theta\right)}{3}\right]_{\theta_{1}}^{\theta_{2}}$.

\section{References}

Alméras T, Clair B (2016) Critical review on the mechanisms of maturation stress generation in trees. J R Soc Interface 13:20160550

Alméras T, Fournier M (2009) Biomechanical design and long-term stability of trees: Morphological and wood traits involved in the balance between weight increase and the gravitropic reaction. $\mathbf{J}$ Theor Biol 256:370-381

Alméras T, Thibaut A, Gril J (2005) Effect of circumferential heterogeneity of wood maturation strain, modulus of elasticity and radial growth on the regulation of stem orientation in trees. Trees Struct Funct 19:457-467

Alméras T, Yoshida M, Okuyama T (2006) The generation of longitudinal maturation stress in wood is not dependent on diurnal changes in diameter of trunk. J Wood Sci 52:452-455

Alméras T, Derycke M, Jaouen G, Beauchene J, Fournier M (2009) Functional diversity in gravitropic reaction among tropical seedlings in relation to ecological and developmental traits. J Exper Bot 60:4397-4410

Archer RR (1986) Growth stresses and strains in trees. Springer, New York

Bastien R, Bohr T, Moulia B, Douady S (2013) Unifying model of shoot gravitropism reveals proprioception as a central feature of posture control in plants. Proc Natl Acad Sci 110(2):755-760 
Clair B, Thibaut B (2014) Physical and Mechanical Properties of Reaction Wood. In: Gardiner B, Barnett J, Saranpää P, Gril J (eds) The biology of reaction wood. Springer, Berlin, pp 171-200

Clair B, Ruelle J, Beauchêne J, Prévost MF, Fournier-Djimbi M (2006) Tension wood and opposite wood in 21 tropical rain forest species. 1. Occurrence and efficiency of the G-layer. IAWA J 27:329-338

Clair B, Alméras T, Pilate G, Jullien D, Sugiyama J, Riekel C (2011) Maturation stress generation in poplar tension wood studied by synchrotron radiation microdiffraction. Plant Physiol 155:562-570

Clair B, Alteyrac J, Gronvold A, Espejo J, Chanson B, Alméras T (2013) Patterns of longitudinal and tangential maturation stresses in Eucalyptus nitens plantation trees. Ann For Sci 70:801-811

Coutand C, Fournier M, Moulia B (2007) The gravitropic response of poplar trunks: key roles of prestressed wood regulation and the relative kinetics of cambial growth versus wood maturation. Plant Physiol 144(2):1166-1180

Coutand C, Pot G, Badel E (2014) Mechanosensing is involved in the regulation of autostress levels in tension wood. Trees 28(3):687-697

Fisher JB, Stevenson JW (1981) Occurence of reaction wood in branches of dicotyledons and its role in tree architecture. Bot Gaz 142:82-95

Fournier M, Chanson B, Thibaut B, Guitard D (1994) Mesures des déformations résiduelles de croissance à la surface des arbres, en relation avec leur morphologie. Observations sur différentes espèces. Ann Sci Forest 51:249-266

Fournier M, Stokes A, Coutand C, Fourcaud T, Moulia B (2006) Tree biomechanics and growth strategies in the context of forest functional ecology. In: Herrel A, Speck T, Rowe N (eds) Ecology and biomechanics: a biomechanical approach of the ecology of animals and plants. CRC Taylor and Francis, Boca Raton, FL, pp 1-33

Fournier M, Dlouha J, Jaouen G, Alméras T (2013) Integrative biomechanics for tree ecology: beyond wood density and strength. J Exp Bot 64(15):4793-4815

Gorshkova T, Mokshina N, Chernova T, Ibragimova N, Salnikov V, Mikshina P, Tryfona T, Banasiak A, Immerzeel P, Dupree P et al
(2015) Aspen tension wood fibers contain beta- $(1 \longrightarrow$ 4)-galactans and acidic arabinogalactans retained by cellulose microfibrils in gelatinous walls. Plant Physiol 169:2048-2063

Huang YS, Hung LF, Kuo-Huang LL (2010) Biomechanical modeling of gravitropic response of branches: roles of asymmetric periphery growth strain versus self-weight bending effect. Trees 24(6):1151-1161

Kikata Y (1972) The effect of lean on growth stress in Pinus densiflora. Mokuzai Gakkaishi 18:443-449

Kubler H (1987) Growth stresses in trees and related wood properties. Forest Abstracts 48:131-189

Lafarguette F, Leplé JC, Déjardin A, Laurans F, Costa G, LesageDescauses MC (2004) Poplar genes encoding fasciclin-like arabinogalactan proteins are highly expressed in tension wood. New Phytol 164:107-121

Moulia B, Coutand B, Lenne C (2006) Posture control and skeletal mechanical acclimation in terrestrial plants: implications for mechanical modeling of plant architecture. Am J Bot 93:1477-1489

Nishikubo N, Awano T, Banasiak A, Bourquin V, Ibatullin F, Funada R, Brumer H, Teeri TT, Hayashi T, Sundberg B et al (2007) Xyloglucan endo-transglycosylase (XET) functions in gelatinous layers of tension wood fibers in Poplar-A glimpse into the mechanism of the balancing act of trees. Plant Cell Physiol 48:843-855

Pilate G, Déjardin A, Laurans F, Leplé JC (2004) Tension wood as a model for functional genomics of wood formation. New Phytol 164:63-72

Roussel JR, Clair B (2015) Evidence of the late lignification of the G-layer in Simarouba tension wood, to assist understanding how non-G-layer species produce tensile stress. Tree Physiol 35(12):1366-1377

Ruelle J, Yamamoto H, Thibaut B (2007) Growth stresses and cellulose structural parameters in tension and normal wood from three tropical rainforest angiosperm species. BioResources 2(2):235-251

Yoshida M (2002) Techniques for measuring growth stress on the xylem surface using strain and dial gauges. Holzforschung 56(5):461-467 